\title{
PERFORMANCE OF GNSS CARRIER-TRACKING LOOP BASED ON KALMAN FILTER IN A CHALLENGING ENVIRONMENT
}

\author{
Yiran Luo ${ }^{1,2,3, *}$, Chunyang $\mathrm{Yu}^{3}, \mathrm{Jian} \mathrm{Li}^{1,2}$, Naser El-Sheimy ${ }^{3}$ \\ ${ }^{1}$ Radar Research Lab, School of Information and Electronics, Beijing Institute of Technology, Beijing, China - \\ yiran.luo@ucalgary.ca, lijian_551@bit.edu.cn \\ ${ }^{2}$ Key Laboratory of Electronic and Information Technology in Satellite Navigation (Beijing Institute of Technology), \\ Ministry of Education, Beijing, China \\ ${ }^{3}$ Department of Geomatics Engineering, University of Calgary, Calgary, Canada - chunyang.yu@ucalgary.ca, elsheimy@ucalgary.ca
}

Commission VI, WG VI/4

KEY WORDS: Global Navigation Satellite System (GNSS), Carrier Tracking Loop, Extended Kalman Filter (EKF), High Dynamics, Challenging Environment, Innovation sequence

\begin{abstract}
:
The global navigation satellite system (GNSS) recently plays an extremely important role in positioning, navigation, and timing (PNT) applications for the modernized automations and mechanizations, e.g., unmanned aerial vehicles (UAVs), unmanned ground vehicles (UGVs), military aircrafts, etc. Nevertheless, GNSS signals are very vulnerable to the influence of various interferences when they are received on Earth, and the reason why it happens is that the long line-of-sight (LOS) distance between the satellite and the receiver user dramatically reduces the power strength after the signal reaches at the ground. The weak GNSS signal is hard to be handled with traditional phase lock loop (PLL), especially in a dynamic environment. Again, the trade-off among the coherent integration time of tracking loop, received signal power strength, and signal or user receiver dynamics is still a tough and remained problem to be solved. The Kalman filter (KF) is always a promising tool to efficiently decrease the random noise for the tracking process. In our work, we evaluate the performances of the tracking loop modelled with both standard KF and extended Kalman filter (EKF). An adaptive algorithm for the covariance matrix of the process noise is contained in our system to increase the tracking ability in a weak and dynamic environment. Besides, a noise channel is also contained to automatically adjust the priori measurement covariance for the KF tracking loop model. Simulation results demonstrate the performance with the proposed technique.
\end{abstract}

\section{INTRODUCTION}

The advent of modernized and new global navigation satellite systems (GNSS), including BeiDou II\&III systems developed by the Chinese government, GPS modernization of the United States (US), and GALILEO satellite navigation system of the European Union (EU), have enhanced their applications in many situations based on positioning, navigation, and timing (PNT), e.g., unmanned aerial vehicles (UAVs) (Gonçalves and Henriques, 2015, Daakir et al., 2017), unmanned ground vehicles (UGVs) (Sivaneri and Gross, 2018), manned aircrafts (Kamel, 2011), some deep space applications (Capuano et al., 2015), etc. If the GNSS receiver works in a weak and dynamic situation, signals received from the navigation satellites would be very hard to be handled with traditional tracking loop. In order to enhance the availability of the GNSS receiver for modern navigation applications, it is important to improve the tracking sensitivity of weak and dynamic GNSS signals.

Various algorithms have been investigated to process the challenging GNSS signals based on previous researches - The maximum likelihood estimation (MLE) method is proposed to estimate the code phase error, carrier phase error, and Doppler shift error in a highly dynamic environment (Won et al., 2012), by which more accurate local replicas can be obtained with the numerically controlled oscillator (NCO). The discrete chirp-Fourier transform (DCFT) is also presented to estimate the Doppler frequency shift and Doppler shift rate of the incoming GNSS signal

\footnotetext{
*Corresponding author
}

(Fan et al., 2013). The FRFT algorithms is reported to estimate the Doppler rate of GNSS signal in the acquisition process (Luo et al., 2018b, Luo et al., 2019) by which the high-dynamic signal can be tracked in a more efficient way with the aiding of the priori Doppler rate estimation (Luo et al., 2018a). Besides, a variational Bayesian adaptive cubature Kalman filter is also exploited to improve the tracking accuracy of high-dynamic GNSS signals (Miao et al., 2017). An improved vector tracking technique has been reported to deal with the high-dynamic tracking in terms of the GNSS carrier phase in recent times (Chen and Gao, 2019). Furthermore, a GNSS signal processing technique with the openloop tracking architecture has been presented to enhance the performance of the GNSS software-defined receiver (SDR) navigation in a challenging urban area (Ruan et al., 2015). In addition, the wavelet transform technique is also evaluated on the improvement for the carrier phase tracking in an extreme situation where the signal power is drastically declined by the ionospheric scintillation interference (Ruan et al., 2017). It can be noticed that the Kalman filter (KF) tracking loops have been involved in most of the previous challenging cases, and this type of tracking architecture plays a significant role in the GNSS-challenging environment for GNSS signal processing.

The main objective of this project is to verify the performance of adaptive KF loops towards the GNSS receiver design in an extreme challenging environment. Two types of KF tracking loops, i.e., standard KF and extended KF (EKF), with self-adaptive algorithms will be investigated to cope with the weak GNSS signal in a highly dynamic environment in this research. At first, 
the system models of the standard KF and EKF tracking loops would be illustrated, respectively. Then, methods to build up the process noise matrix and measurement noise matrix will be described, where the process noise model has been improved with a self-adaptive technique based on the innovation sequence, and the measurement noise matrix is automatically adjusted with the thermal noise jitter model of the two-quadrant arctangent carrier discriminator based on an extra noise tracking channel. Finally, a highly dynamic signal model which was defined by the American jet propulsion laboratory (JPL) (Vilnrotter et al., 1988) will be used to verify the performance of the proposed algorithms when the input carrier-to-noise ratio density $\left(C / N_{0}\right)$ is decided in different levels.

\section{GNSS CARRIER TRACKING LOOPS BASED ON KALMAN FILTER}

In this section, the signal carrier tracking loop of GNSS will be modelled with standard KF and EKF, respectively.

\subsection{Standard Kalman Filter Model}

The 4th-order KF tracking loop can estimate the carrier phase error, Doppler angular velocity, Doppler angular acceleration and Doppler angular jerk on condition that the system model can be accurately constructed as well as the priori noise variance can be given. The associated state vector satisfies

$$
\mathbf{x}_{k}=\left[\begin{array}{llll}
\Delta \theta_{k} & \omega_{k} & \alpha_{k} & \beta_{k}
\end{array}\right]^{T}
$$

where $\Delta \theta_{k}$ is the carrier phase error, $\omega_{k}$ denotes the carrier angular frequency, $\alpha_{k}$ represents the carrier angular frequency rate, $\beta_{k}$ stands for the carrier angular jerk. The system state transition equation can be given by

$$
\mathbf{x}_{k}=\boldsymbol{\Phi}_{k, k-1} \mathbf{x}_{k-1}+\mathbf{B}_{k-1} u_{k-1}+\mathbf{w}_{k-1}
$$

with

$$
\begin{gathered}
\boldsymbol{\Phi}_{k, k-1}=\left[\begin{array}{cccc}
1 & \Delta t & \frac{1}{2} \Delta t^{2} & \frac{1}{6} \Delta t^{3} \\
0 & 1 & \Delta t & \frac{1}{2} \Delta t^{2} \\
0 & 0 & 1 & \Delta t \\
0 & 0 & 0 & 1
\end{array}\right] \\
\mathbf{B}_{k-1}=-\Delta t\left[\begin{array}{l}
1 \\
0 \\
0 \\
0
\end{array}\right], u_{k-1}=\left[2 \pi\left(\hat{f}_{k}-f_{i}-f_{0}\right)\right]
\end{gathered}
$$

where $\boldsymbol{\Phi}_{k, k-1}$ stands for the state transition matrix, $\mathbf{B}_{k-1}$ represents the state control matrix, $u_{k-1}$ denotes the control variable, $\mathbf{w}_{k-1}$ is the process noise, $\hat{f}_{k}$ is the local replica of the carrier frequency adjusted by the NCO, and $\Delta t$ stands for the updating time, $f_{i}$ and $f_{0}$ represent the intermediate frequency and initial Doppler frequency, respectively. Next, the system observation equation can be expressed as (Psiaki and Jung, 2002)

$$
z_{k}=\mathbf{H}_{k} \mathbf{x}_{k}+C_{k} u_{k-1}+v_{k}
$$

with

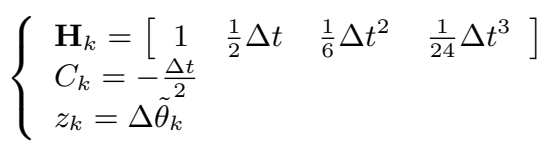

where $\mathbf{H}_{k}$ is the observation vector, $C_{k}$ stands for the observation control value to adjust the systematic error for the measurement in this work, $v_{k}$ denotes the observation noise. Only the carrier phase error, $\Delta \tilde{\theta}_{k}$, offered by the arctangent carrier discriminator is contained in the system as a measurement for each updating interval. Since only the GNSS carrier signal is contained in our subsequent simulations, the bit transition ambiguity will not occur in the tracking process. In this case, the four-quadrant arctangent carrier discriminator is used in this research which can be given by

$$
\Delta \tilde{\theta}_{k}=\operatorname{atan} 2\left(Q_{k}, I_{k}\right)
$$

where $\operatorname{atan} 2(\cdot)$ denotes the four-quadrant arctangent discriminator; $Q_{k}$ and $I_{k}$ represent the quadrature and in-phase components of the outputs with integration and dumping (I\&D) implementation, respectively (Kaplan and Hegarty, 2006). To further explain why the four-quadrant arctangent discriminator is used here, on the one hand, the data code can be wiped off using the A-GPS or A-GNSS technique (Kaplan and Hegarty, 2006) in practical situation; on the other hand, the bit sign is also able to be addressed with the given secondary code in the pilot channel formed in some binary offset carrier (BOC) signals, e.g., GPS L1C (ISGPS-800E, 2018) and Galileo E1 (EU, 2015).

\subsection{Extended Kalman Filter Model}

Referring to previous researches, the carrier tracking loop developed with the EKF algorithm has been attracted more attention than the standard KF one (Petovello and Lachapelle, 2006, Ruan et al., 2017). The state vector for the EKF-based carrier tracking loop can be given by

$$
\delta \mathbf{x}_{k}=\left[\begin{array}{llll}
\delta \Delta \theta_{k} & \delta \omega_{k} & \delta \alpha_{k} & \delta \beta_{k}
\end{array}\right]^{T}
$$

where $\delta \Delta \theta_{k}$ denotes the error of the carrier phase residual, $\delta \omega_{k}$ $\delta \alpha_{k}$, and $\delta \beta_{k}$ represent the carrier angular frequency error, the error of the carrier angular frequency rate, and the error of the carrier angular jerk, respectively. The state transition equation can be expressed as

$$
\delta \mathbf{x}_{k}=\boldsymbol{\Phi}_{k, k-1} \delta \mathbf{x}_{k-1}+\mathbf{w}_{k-1}
$$

and the observation equation is given by

$$
z_{k}=\mathbf{H}_{k} \delta \mathbf{x}_{k}+v_{k}
$$

The transition matrix and the observation matrix are identical to the ones built in standard KF tracking model. 


\subsection{Process and Measurement Covariance Matrices}

2.3.1 Process Covariance Matrix: Ignoring the subscript for the epoch denotation, in this research, the process noise for each of the unknown states follows the discrete Gaussian white noise distribution, and the noise vector is given by

$$
w=\left[w_{b}, w_{d}, 0, w_{j}\right]^{\mathrm{T}}
$$

where $w_{b}$ and $w_{d}$ stand for the normal random walks with zeros means for clock bias and drift, respectively; $w_{j}$ denotes the process noise for the carrier angular jerk (Brown and Hwang, 2012). These noise terms are assumed to be independent with each other. So, the corresponding covariance matrix can be given by

$$
\left\{\begin{array}{l}
E\left[w_{b} w_{b}^{\mathrm{T}}\right]=S_{f} \\
E\left[w_{d} w_{d}^{\mathrm{T}}\right]=S_{g} \\
E\left[w_{j} w_{j}^{\mathrm{T}}\right]=S_{j}
\end{array}\right.
$$

where $E[\cdot]$ is the expectation operator of the variable. Accordingly, the process noise covariance matrix can be obtained as

$$
\mathbf{Q}=\left[\begin{array}{cccc}
S_{f} & 0 & 0 & 0 \\
0 & S_{g} & 0 & 0 \\
0 & 0 & 0 & 0 \\
0 & 0 & 0 & S_{j}
\end{array}\right]
$$

Since the algorithm is working in a discrete-observations-based case, the transition terms for the process noise matrix is finally derived as

$$
\mathbf{Q}_{k}=\int_{0}^{\Delta t} \mathbf{\Phi} \times \mathbf{Q} \times \boldsymbol{\Phi}^{\mathrm{T}} d t
$$

where the transition matrix, $\boldsymbol{\Phi}$, is given by (3); since it is not changed with the time variable, the subscript is omitted here; $S_{f}$ and $S_{g}$ represent the spectral densities of the clock bias error and clock drift error, respectively, and they can be obtained in previous reference (Brown and Hwang, 2012); $S_{j}$ denotes spectral density associated with the white noise caused by the change of Doppler rate or dynamics between the satellite and the receiver along the LOS direction.

2.3.2 Adaptive Measurement Covariance Matrix: Since the arctangent carrier discriminator is used for the carrier tracking loop in our research, the corresponding thermal noise jitter in radian is given as follow (Kaplan and Hegarty, 2006)

$$
\sigma_{\theta}=\sqrt{\frac{B_{p}}{C / N_{0}}\left(1+\frac{1}{2 \Delta t C / N_{0}}\right)}
$$

with $B_{p}=\frac{1}{\Delta t}$, where $B_{p}$ is carrier loop noise bandwidth in $\mathrm{Hz}$, $C / N_{0}$ denotes the carrier-to-noise density ratio in $\mathrm{Hz}$ here, $\Delta t$ stands for the coherent integration time in tracking or the updating interval of the KF-based tracking loop, and the part of the equation involving, $\Delta t$ accounts for the squaring loss. Regardless of the other noise sources and considering the given thermal noise jitter as the distribution of the priori measurement noise, the measurement covariance matrix of the KF-based tracking loop has the access to be automatically adjusted with the $C / N_{0}$ estimation. The value of $C / N_{0}$ will be calculated with an extra noise channel in the system. Therefore, the measurement covariance is given by

$$
R_{k}=\sigma_{\theta}^{2}
$$

2.3.3 Self-Adaptive Algorithm for Process Covariance Matrix: The difference between the latest measurement and a priori estimate is called the innovation sequence, $V_{k}$, which is given as the follow (Salychev, 1998)

$$
\begin{aligned}
V_{k} & =z_{k}-\mathbf{H}_{k} \boldsymbol{\Phi}_{k, k-1} \hat{\mathbf{x}}_{k-1} \\
& =z_{k}-\mathbf{H}_{k} \hat{\mathbf{x}}_{k, k-1} \\
& =\mathbf{H}_{k} \mathbf{x}_{k}+v_{k}-\mathbf{H}_{k} \hat{\mathbf{x}}_{k, k-1} \\
& =\mathbf{H}_{k} \tilde{\mathbf{x}}_{k, k-1}+v_{k}
\end{aligned}
$$

where $\hat{\mathbf{x}}_{k, k-1}$ is the priori estimation, and $\tilde{\mathbf{x}}_{k, k-1}$ is the priori estimation error. Then, covariance matrix of the innovation sequence can be derived as

$$
\Gamma_{k}=E\left(V_{k} V_{k}^{T}\right)=\mathbf{H}_{k} \mathbf{P}_{k, k-1}\left(\mathbf{H}_{k}\right)^{T}+R_{k}
$$

where $\mathbf{P}_{k, k-1}$ represents the priori state covariance matrix.

Since the priori process driving error of the incoming GNSS signal which follows the Gaussian white noise distribution may be contaminated in a practical environment, a self-adaptive algorithm based on the innovation sequence is presented to help improve the performance of carrier tracking. $R_{k}$ is known and the initial process noise covariance is also given by (14). When the $\mathrm{KF}$ tracking loop is working, $\mathbf{Q}_{k}$ will be influenced by the practical tracking results. Given

$$
\begin{aligned}
& \hat{\mathbf{x}}_{k}-\boldsymbol{\Phi}_{k, k-1} \hat{\mathbf{x}}_{k-1} \\
& =\hat{\mathbf{x}}_{k, k-1}+\mathbf{K}_{k}\left(z_{k}-\mathbf{H}_{k} \hat{\mathbf{x}}_{k, k-1}\right)-\boldsymbol{\Phi}_{k, k-1} \hat{\mathbf{x}}_{k-1} \\
& =\hat{\mathbf{x}}_{k, k-1}+\mathbf{K}_{k}\left(z_{k}-\mathbf{H}_{k} \hat{\mathbf{x}}_{k, k-1}\right)-\hat{\mathbf{x}}_{k, k-1} \\
& =\mathbf{K}_{k}\left(z_{k}-\mathbf{H}_{k} \hat{\mathbf{x}}_{k, k-1}\right) \\
& =\mathbf{K}_{k} V_{k}
\end{aligned}
$$

Then, it can be given by

$$
\left\{\begin{array}{r}
\hat{\mathbf{x}}_{k}-\boldsymbol{\Phi}_{k, k-1} \hat{\mathbf{x}}_{k-1}=\hat{\mathbf{w}}_{k-1} \\
\hat{\mathbf{x}}_{k}-\boldsymbol{\Phi}_{k, k-1} \hat{\mathbf{x}}_{k-1}=\mathbf{K}_{k} V_{k}
\end{array}\right.
$$

Therefore,

$$
\hat{\mathbf{Q}}_{k-1}^{-}=\mathbf{K}_{k} E\left(V_{k} V_{k}^{T}\right) \mathbf{K}_{k}^{T}
$$

Assuming that $\mathbf{K}_{k} \approx \mathbf{K}_{k-1}$ and $\mathbf{P}_{k, k-1} \approx \mathbf{P}_{k-1}$, equations (19) and (21) can be rewritten as

$$
\left\{\begin{array}{l}
\hat{\Gamma}_{k} \approx \mathbf{H}_{k} \mathbf{P}_{k-1}\left(\mathbf{H}_{k}\right)^{T}+R_{k} \\
\hat{\mathbf{Q}}_{k-1}^{-} \approx \mathbf{K}_{k-1} \hat{\Gamma}_{k}\left(\mathbf{K}_{k-1}\right)^{T}
\end{array}\right.
$$


In practical implementation, the $\alpha-\beta$ filtering algorithm is used to smooth the self-adaptive variance matrix of process noise whose initial value is determined with (14). The final process covariance matrix can be given by

$$
\hat{\mathbf{Q}}_{k-1}=\alpha \cdot \hat{\mathbf{Q}}_{k-2}+\beta \cdot \hat{\mathbf{Q}}_{k-1}^{-}
$$

where $\alpha$ is chosen as $\frac{511}{512}$ and $\beta$ is set to $\frac{1}{512}$ in this work.

Therefore, the self-adaptive algorithm for the process covariance matrix is done, and the unknown states can be estimated with the KF recursive formula (Faragher, 2012).

\section{CARRIER NCO UPDATES}

Updating processes for the carrier NCO of the tracking loop differ in different KF-based tracking architectures.

\subsection{Standard Kalman Filter Carrier Tracking Loop:}

The carrier frequency NCO based on the standard KF tracking loop can be updated as the follow

$$
\begin{aligned}
& \hat{f}_{k+1}=\left(f_{i}+f_{0}\right) \\
& +\frac{1}{2 \pi \Delta t}\left(\Delta \hat{\theta}_{k}+\hat{\omega}_{k} \Delta t+\frac{1}{2} \hat{\alpha}_{k} \Delta t^{2}+\frac{1}{6} \hat{\beta}_{k} \Delta t^{3}\right)
\end{aligned}
$$

with

$$
\hat{\mathbf{x}}_{k}=\left[\begin{array}{llll}
\Delta \hat{\theta}_{k} & \hat{\omega}_{k} & \hat{\alpha}_{k} & \hat{\beta}_{k}
\end{array}\right]^{T}
$$

where $\hat{\mathbf{x}}_{k}$ is estimated with the standard KF method.

\subsection{Extended Kalman Filter Carrier Tracking Loop:}

The residual of carrier phase error is estimated with the EKF approach during each updating interval. Accordingly, the unknown state variables related to the NCO update are estimated by

$$
\hat{\mathbf{x}}_{k}=\hat{\mathbf{x}}_{k-1}+\delta \hat{\mathbf{x}}_{k}
$$

which means, the associated residual can be firstly derived as

$$
\begin{aligned}
& \delta \hat{f}_{k}=\frac{1}{2 \pi \Delta t} \\
& \times\left(\delta \Delta \hat{\theta}_{k}+\delta \hat{\omega}_{k} \Delta t+\frac{1}{2} \delta \hat{\alpha}_{k} \Delta t^{2}+\frac{1}{6} \delta \hat{\beta}_{k} \Delta t^{3}\right)
\end{aligned}
$$

Finally, the NCO of the carrier frequency can be obtained as

$$
\begin{aligned}
& \hat{f}_{k+1}=\left(f_{i}+f_{0}\right)+\delta \hat{f}_{k}+\frac{1}{2 \pi \Delta t} \\
& \times\left(\Delta \hat{\theta}_{k-1}+\hat{\omega}_{k-1} \Delta t+\frac{1}{2} \hat{\alpha}_{k-1} \Delta t^{2}+\frac{1}{6} \hat{\beta}_{k-1} \Delta t^{3}\right)
\end{aligned}
$$

Therefore, the carrier frequency NCO is controlled by $\hat{f}_{k+1}$ based on standard KF or EKF methods within the updating interval in our work. The process covariance matrix and measurement covariance matrix are built in the same way for these two approaches, and both can be adaptively adjusted depending on the real-time estimation results.

\section{SIMULATIONS AND EXPERIMENT RESULTS}

In this section, both carrier tracking loop modelled with standard KF and EKF architectures with proposed self-adaptive algorithms will be tested in simulations. Matlab R2016b software will be used in the experiments to generate the GNSS carrier signals formed with a typical high-dynamic model which will be subsequently described. Simulation experiment results demonstrate the performances of the carrier tracking loop based on the adaptive algorithms for the KF covariance matrices.

The related clock spectral densities are initialized in accordance with the given coefficients for OCXO as listed in Table 9.1 of this provided reference (Brown and Hwang, 2012); the numerical value for $S_{j}$ is set to $\left(2 \pi \times 1000 \times f_{r} / c\right)^{2}$ allowing the $\mathrm{KF}$ carrier tracking loop to tolerate the dynamic model which will be subsequently described and used in the simulations, where $f_{r}$ is the radio frequency of the GPS L1 C/A signal, and $c$ denotes the speed of the light. The process covariance matrix and the associated coefficients are highly related to the bandwidth value of the carrier loop formed with the KF algorithms.

\subsection{High-Dynamic Model Applied in Simulations}

In simulations, the highly dynamic signal model was defined by the American JPL, and Figure 1 illustrates the model, where $g$ denotes the gravity. The motion can be depicted as Table 1. In this section, the standard KF and EKF tracking loop with proposed self-adaptive algorithms are both simulated to track the high-dynamic GNSS signal carrier under the condition that the $C / N_{0}$ ranges from $25 \mathrm{~dB}-\mathrm{Hz}$ to $45 \mathrm{~dB}-\mathrm{Hz}$. The coherent integration time, which can be also regarded as the updating interval for $\mathrm{KFs}$, is set as $5 \mathrm{~ms}$ in simulations.
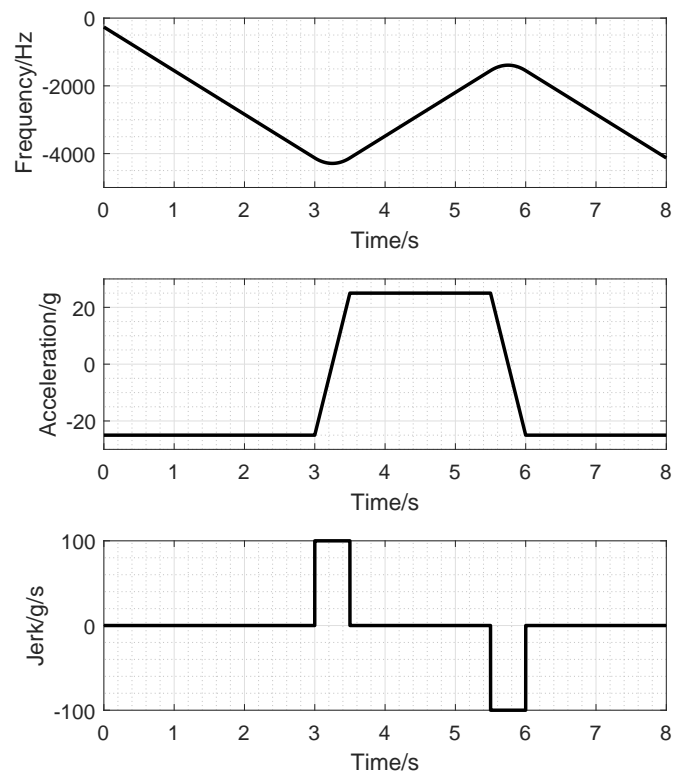

Figure 1 . Highly dynamic signal model.

\subsection{Phase Lock Indicator}

The phase lock indicator (PLI) is used to evaluate the tracking performance of the signal carrier in simulations. The PLI was 


\begin{tabular}{|c|c|c|}
\hline Time & $0 \mathrm{~s}$ & The initial speed is $-50 \mathrm{~m} / \mathrm{s} ;$ \\
& $0 \mathrm{~s}-3 \mathrm{~s}$ & The acceleration is $-25 \mathrm{~g} ;$ \\
& $3 \mathrm{~s}-3.5 \mathrm{~s}$ & Add the jerk of $100 \mathrm{~g} / \mathrm{s} ;$ \\
& $3.5 \mathrm{~s}-5.5 \mathrm{~s}$ & The acceleration increases to $25 \mathrm{~g} ;$ \\
& $5.5 \mathrm{~s}-6 \mathrm{~s}$ & Add the jerk of $-100 \mathrm{~g} / \mathrm{s} ;$ \\
$6 \mathrm{~s}-8 \mathrm{~s}$ & The acceleration decreases to $-25 \mathrm{~g}$. \\
\hline
\end{tabular}

Table 1 . The description of the highly dynamic motion.

presented by Salem et al. in 2012 (Salem et al., 2012), and it is given as the follow

$$
P L I \approx \cos (2 \Delta \theta)
$$

The value of PLI is expected to range from -1 to 1 . When the PLI reaches to -1 , the local replica of the GNSS signal is completely out of lock with the incoming signal, while 1 indicates a perfect match.

\subsection{Simulation Results}

Four types of KF tracking methods are involved in the experiments, i.e., tracking loop based on standard KF method, EKF method, standard KF with self-adaptive process variance matrix, EKF with self-adaptive process variance matrix, and these four methods are associated with the short forms of SKF, EKF, A-SKF, A-EKF, respectively. The self-adaptive process variance matrix is calculated with (23).

Simulation experiments have been carried out in the situations where the $C / N_{0}$ ranges from $25 \mathrm{~dB}-\mathrm{Hz}$ to $45 \mathrm{~dB}-\mathrm{Hz}$. The rootmean-square error (RMSE) results based on four KF-based carrier tracking loops are illustrated in Figure 2. Furthermore, the respective means and RMSEs of the PLI are shown in Figure 3 and Figure 4. Some conclusions can be drawn from the simulation results.

At first, as the input $C / N_{0}$ decreases, the KF tracking loop based on the self-adaptive process variance matrix is more promising than the ordinary ones to replicate the local carrier signals. More tracking noise has been reduced when the tracking loop works in a weak and dynamic environment.

However, when the input $C / N_{0}$ is higher than around $39 \mathrm{~dB}$ $\mathrm{Hz}$, the self-adaptive algorithms does not perform better than the ordinary KF algorithms any more as illustrated in Figure 2. PLI curves provided in Figure 5 also proves that the performances of self-adaptive KF tracking loop outperform the ones with the ordinary KF tracking algorithm when they are working in a weak situation while the former performs worse than the latter when the incoming signal power is in a good condition.

Although a large amount of the tracking noise has been decreased with the self-adaptive KF algorithms during the tracking time spanning, more time will be taken by the tracking loop to sense the change of the incoming dynamics. For example, the tracking errors of the ordinary standard KF/EKF-based algorithms become larger than the ones with self-adaptive methods at the time when the incoming jerks occur. In addition, it can be noticed that the PLI performance differences between the adaptive EKF and the ordinary EKF shrink regarding the results based on the standard $\mathrm{KF}$ algorithms as the comparisons. Estimation results of PLI in Figure 3 and Figure 4, tracking results in Figure 6 and Figure 7 could demonstrate this conclusion.
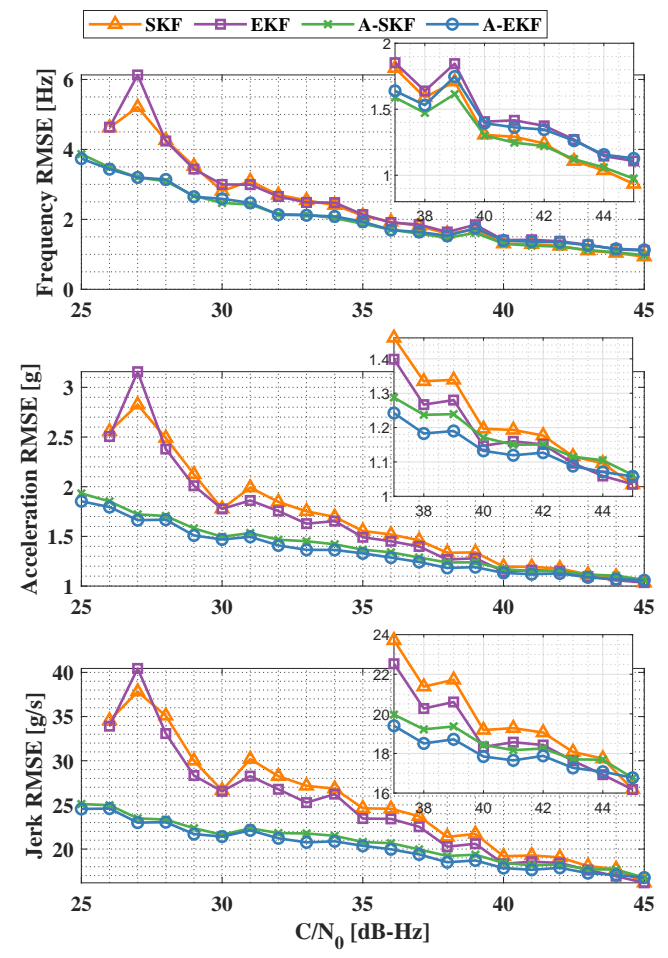

Figure 2. RMSEs of carrier tracking errors.

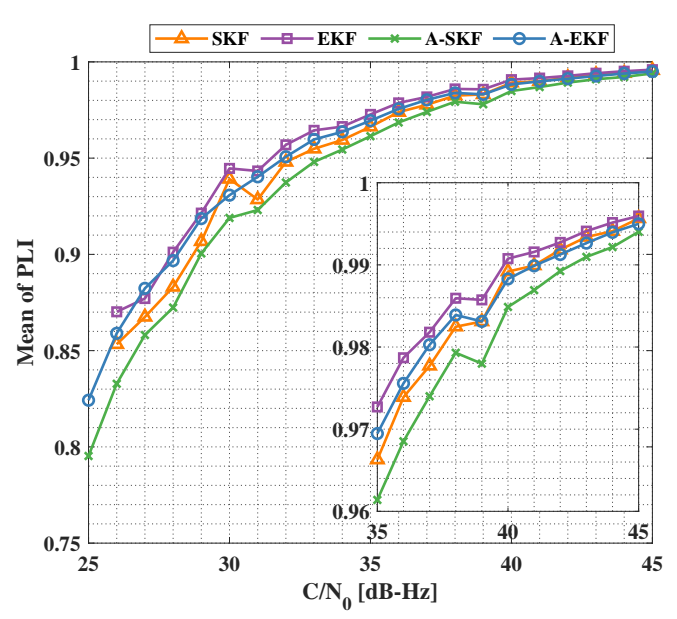

Figure 3. Means of the PLI.

Then, it has also been verified that the tracking results based on EKF algorithms are slightly better than the results with standard $\mathrm{KF}$ tracking loops especially when the signal is very weak. Nevertheless, when the input $C / N_{0}$ of the received signal increases, the tracking frequency errors with the standard KF are slightly smaller than the errors of EKF algorithms while experiment results with the EKF in acceleration error and jerk error have been proved to be a bit smaller. The tested PLI errors of these two algorithms are very close to each other. Furthermore, the RMSE of PLI with standard KF perform a bit better than the ones with EKF algorithms, while the conclusions in terms of the mean of PLI is opposite.

Besides, the tracking sensitivity has been enhanced regarding that the tracking loop with self-adaptive algorithms manage to ob- 


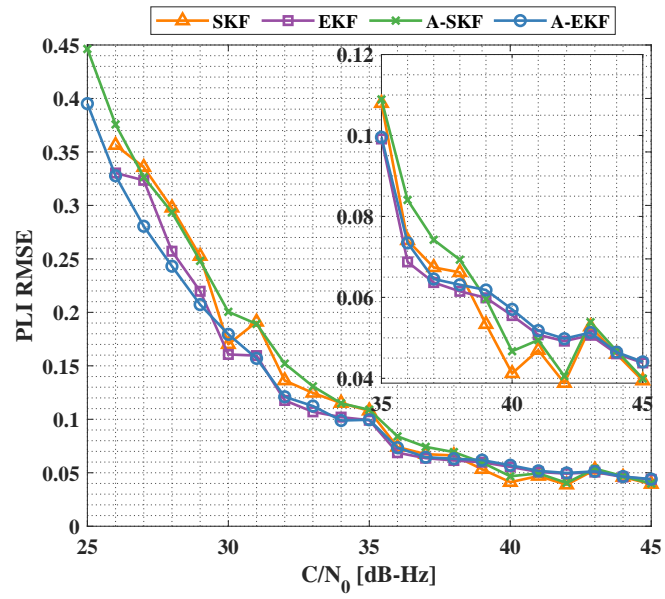

Figure 4. RMSEs of the PLI.

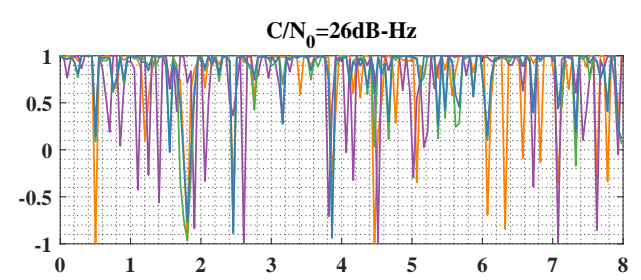

$\mathrm{C} / \mathrm{N}_{0}=45 \mathrm{~dB}-\mathrm{Hz}$

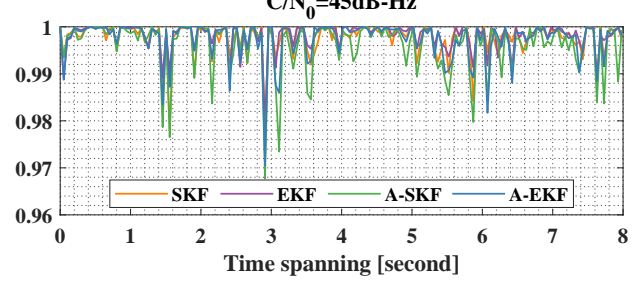

Figure 5. PLI estimation results.

tain the local replicas in a $25-\mathrm{dB}-\mathrm{Hz}$ situation while it fails to be done using KF-based algorithms without self-adaptive algorithms. Corresponding tracking results are given in Figure 8.

In the end, the carrier tracking results and errors for the high dynamic signal model in the environment where the input $C / N_{0}$ is $26 \mathrm{~dB}-\mathrm{Hz}$ are illustrated in Figure 6 and Figure 7.

\section{CONCLUSIONS}

We discussed the performances of the carrier tracking loops based on four types KF algorithms, i.e., standard KF and EKF with selfadaptive process variance matrix, standard KF and EKF without such self-adaptive approach, in a weak and dynamic environment. Experiment results have proved that the adaptive algorithms for both standard KF and EKF tracking architectures can enhance the tracking performance in a challenging environment, e.g., the accuracies of tracking error as well as the tracking sensitivities have been improved in a typical dynamic situation, especially when the incoming signal power is very weak. On the other hand, it has been verified that the EKF algorithms slightly outperform the standard $\mathrm{KF}$ one in a challenging environment. However, as the input $C / N_{0}$ increases to around $39 \mathrm{~dB}-\mathrm{Hz}$, tracking performances for these two KFs would be very close to each other. The results based on standard KF approach can even become slightly
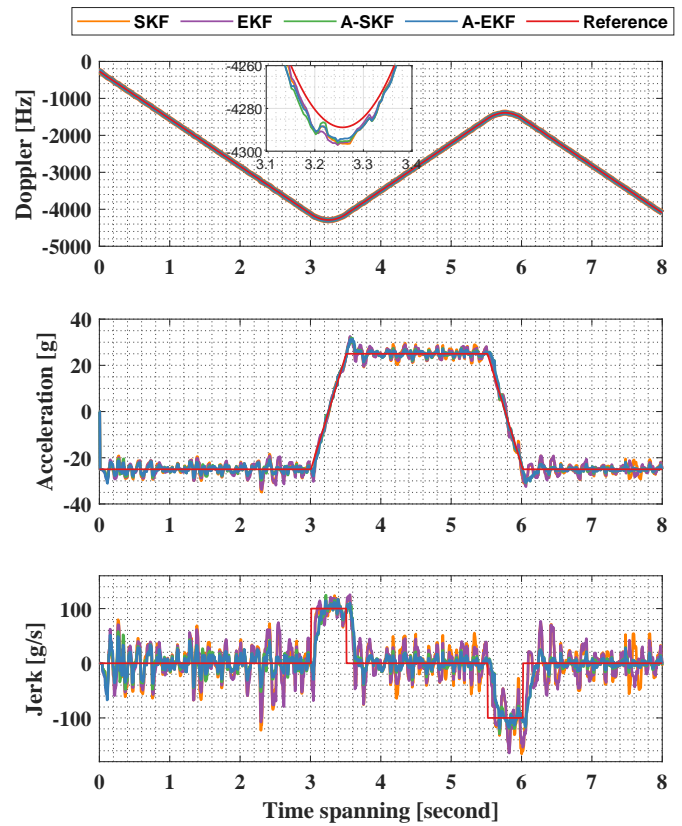

Figure 6. Tracking results when $\mathrm{C} / N_{0}$ is set to $26 \mathrm{~dB}-\mathrm{Hz}$.
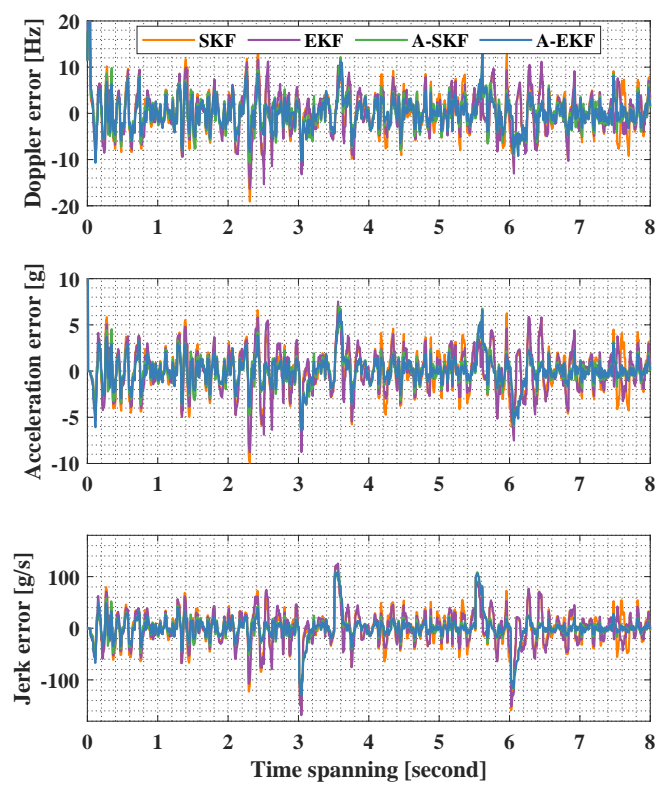

Figure 7. Tracking errors when $\mathrm{C} / N_{0}$ is set to $26 \mathrm{~dB}-\mathrm{Hz}$.

better than the EKF-based ones in the situation when the receiver signal power is stronger.

\section{ACKNOWLEDGEMENTS}

This work was supported in part by the National Natural Science Foundation of China under Grant 31727901, and in part by the Chang Jiang Scholars Programme under Grant T2012122. 

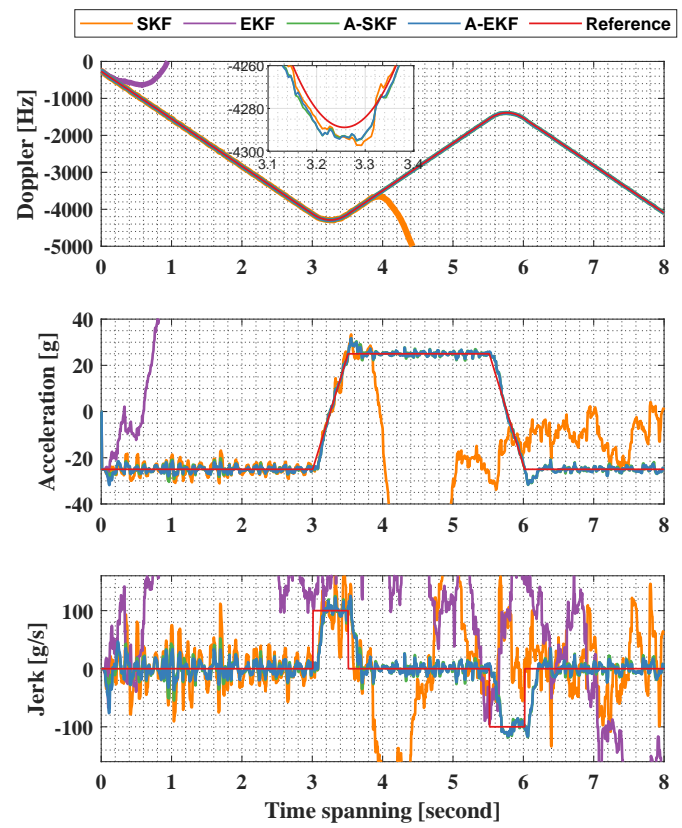

Figure 8. Tracking results when $\mathrm{C} / N_{0}$ is set to $25 \mathrm{~dB}-\mathrm{Hz}$.

\section{REFERENCES}

Brown, R. G. and Hwang, P. Y. C., 2012. Introduction to random signals and applied Kalman filter with Matlab exercises. 4 edn, Wiley, Hoboken.

Capuano, V., Botteron, C., Leclère, J., Tian, J., Wang, Y. and Farine, P. A., 2015. Feasibility study of GNSS as navigation system to reach the Moon. Acta Astronautica 116, pp. 186-201.

Chen, S. and Gao, Y., 2019. Improvement of carrier phase tracking in high dynamics conditions using an adaptive joint vector tracking architecture. GPS Solutions 23(1), pp. 15.

Daakir, M., Pierrot-Deseilligny, M., Bosser, P., Pichard, F., Thom, C., Rabot, Y. and Martin, O., 2017. Lightweight UAV with on-board photogrammetry and single-frequency GPS positioning for metrology applications. ISPRS Journal of Photogrammetry and Remote Sensing 127, pp. 115-126.

EU, 2015. European GNSS (Galileo) Open Service Signal In Space Interface Control Document. Office for Official Publications of the European Communities.

Fan, B., Zhang, K., Qin, Y., Wang, H. and Xiao, H., 2013. Discrete chirp-Fourier transform-based acquisition algorithm for weak global positioning system L5 signals in high dynamic environments. IET Radar, Sonar \& Navigation 7(7), pp. 736-746.

Faragher, R., 2012. Understanding the Basis of the Kalman Filter Via a Simple and Intuitive Derivation [Lecture Notes]. IEEE Signal Processing Magazine 29(5), pp. 128-132.

Gonçalves, J. A. and Henriques, R., 2015. UAV photogrammetry for topographic monitoring of coastal areas. ISPRS Journal of Photogrammetry and Remote Sensing 104, pp. 101-111.

IS-GPS-800E, 2018. Global Positioning Systems Directorate Systems Engineering and Integration Interface Speccification IS-GPS-800E. Technical report, Navstar GPS Space Segment/Navigation User Interfaces.

Kamel, A. M. M., 2011. Context aware high dynamics GNSSINS for interference mitigation [Ph. D. thesis]. Ph. d. thesis, University of Calgary.
Kaplan, E. D. and Hegarty, C. J., 2006. Understanding GPS: Principles and Applications Second Edition. Artech house.

Luo, Y., Yu, C., Chen, S., Li, J., Ruan, H. and El-Sheimy, N., 2019. A Novel Doppler Rate Estimator Based on Fractional Fourier Transform for High-Dynamic GNSS Signal. IEEE Access 7 , pp. $29575-29596$.

Luo, Y., Zhang, L. and El-Sheimy, N., 2018a. An improved de-kfl for boc signal tracking assisted by frft in a highly dynamic environment. In: 2018 IEEE/ION Position, Location and Navigation Symposium (PLANS), pp. 1525-1534.

Luo, Y., Zhang, L. and Ruan, H., 2018b. An Acquisition Algorithm Based on FRFT for Weak GNSS Signals in A Dynamic Environment. IEEE Communications Letters 22(6), pp. 12121215.

Miao, Z.-y., Lv, Y.-1., Xu, D.-j., Shen, F. and Pang, S.-w., 2017. Analysis of a variational bayesian adaptive cubature kalman filter tracking loop for high dynamic conditions. GPS Solutions 21(1), pp. 111-122.

Petovello, M. and Lachapelle, G., 2006. Comparison of VectorBased Software Receiver Implementations With Application to Ultra-Tight GPS/INS Integration. In: Proceedings of the 19th International Technical Meeting of the Satellite Division of The Institute of Navigation (ION GNSS 2006), Fort Worth, TX, pp. 1790-1799.

Psiaki, M. L. and Jung, H., 2002. Extended Kalman filter methods for tracking weak GPS signals. In: Proceedings of the 15th International Technical Meeting of the Satellite Division of The Institute of Navigation (ION GPS 2002), Portland, OR, pp. 25392553.

Ruan, H., Li, J., Zhang, L. and Long, T., 2015. Adaptive correlation space adjusted open-loop tracking approach for vehicle positioning with global navigation satellite system in urban areas. Sensors (Switzerland) 15(9), pp. 21581-21612.

Ruan, H., Zhang, L., Luo, Y. and Long, T., 2017. GNSS Carrier Phase Tracking With Discrete Wavelet Transform Filtering Under Ionospheric Scintillation. IEEE Communications Letters 21(2), pp. 394-397.

Salem, D. R., O'Driscoll, C. and Lachapelle, G., 2012. Methodology for comparing two carrier phase tracking techniques. GPS Solutions 16(2), pp. 197-207.

Salychev, O. S., 1998. Inertial Systems in Navigation and Geophysics. Moscow: Bauman MSTU Press.

Sivaneri, V. O. and Gross, J. N., 2018. Flight-testing of a cooperative UGV-to-UAV strategy for improved positioning in challenging GNSS environments. Aerospace Science and Technology 82-83, pp. 575-582.

Vilnrotter, V., Hinedi, S. and Kumar, R., 1988. A Comparision of Frequency Estimation Techniques for High-Dynamic Trajectories. JPL Publication 88.

Won, J.-H., Pany, T. and Eissfeller, B., 2012. Iterative Maximum Likelihood Estimators for High-Dynamic GNSS Signal Tracking. IEEE Transactions on Aerospace and Electronic Systems 48(4), pp. 2875-2893. 